\title{
A Bi-normalized Frequency Estimation Algorithm
}

\author{
Zhaobi Chu, Yan Wang and Rui Zhang \\ School of Electrical Engineering and Automation ,Hefei University of Technology ,Hefei 230009,China
}

\begin{abstract}
This paper presents a bi-normalized frequency estimation algorithm which contains a two-dimensional state estimation equation and a one-dimensional frequency update rule coupled with it. The robustness of the algorithm is that the frequency estimation convergence is no longer subject to the actual value of the amplitude and frequency of the estimated signal. The asymptotic convergence of frequency estimation is demonstrated by Lyapunov stability theory, perturbation method, integral manifold and Mathieu equation, and the relationship between adaptive internal model algorithm and the proposed bi-normalization algorithm is analyzed. Simulation results verify the effectiveness of the proposed algorithm.
\end{abstract}

Keywords - frequency estimation; adaptive internal model; bi-normalization; robustness

\section{INTRODUCTION}

In harmonic detection and interference suppression of signals, frequency estimation has always been one of the core contents of the research. At present, there are a large number of methods for estimating the frequency of periodic signals, such as adaptive notch filtering algorithm ${ }^{[1-4]}$, the combination of internal model controller and adaptive algorithm ${ }^{[5-7]}$, phase-locked loop ${ }^{[8]}$, the second-order generalized integrator ${ }^{[9]}$, the combination of the second-order generalized integrator and the frequency-locked loop ${ }^{[10]}$, etc. These algorithms have been appropriately adjusted and improved for different models and application backgrounds, enriching solutions to the estimation of the periodic signal frequency. In addition, in order to eliminate the influence of amplitude, a normalized way is often used. At present, most of the proposed normalization methods ${ }^{[15-16]}$ are to eliminate the influence of amplitude on the convergence speed of the algorithm.

According to [1], a globally converged adaptive filtering algorithm for estimating the unknown frequency of a signal online was proposed. Then based on the above paper, a new algorithm which provided the range of parameters for convergent system was presented in [6] .Later theoretical Analysis of System Stability in the Range of Parameters was raised in [12]. The proposed adaptive internal model algorithm combines the internal model structure with an adaptive algorithm to eliminate the perturbation of periodic signals when performing frequency estimation.

In order to degrade the effect of frequency on the convergence of the algorithm and give the parameter range of system stability, we develop a bi-normalized frequency estimation algorithm. The state space equation of this scheme, the problem statement and the proposed algorithm is presented in section 2 of this paper. At the same time, the performance of the algorithm is analyzed, and the reference parameter range to ensure system convergence is obtained in Section 3. Section 4 shows simulations to verify the performance of the proposed algorithm.

\section{Problem STATEMENT}

In Adaptive internal model algorithm ${ }^{[7]}$,input signal choose : $y(\tau)=A_{0} \sin \left(\omega_{0} \tau+\delta_{0}\right), A_{0}, \omega_{0}, \delta_{0}$ is unknown and the state equations are:

$$
\begin{aligned}
& \int \frac{d x_{1}}{d \tau}(\tau)=-\hat{\omega}(\tau) \widehat{x}_{2}(\tau) \\
& \left\{\frac{d \widehat{x}_{2}}{d \tau}(\tau)=\hat{\omega}(\tau) \widehat{x}_{1}(\tau)+K_{f} e\right. \\
& \frac{d \widehat{\omega}}{d \tau}(\tau)=K_{e} \frac{K_{f} e \widehat{x}_{1}(\tau)}{\hat{x}_{1}^{2}(\tau)+\hat{x}_{2}^{2}(\tau)} \\
& e=y-x_{2} \\
& \hat{A}(\tau)=\sqrt{x_{1}^{2}(\tau)+x_{2}^{2}(\tau)}
\end{aligned}
$$

In which, ()$^{\prime}=\mathrm{d}() / \mathrm{d} \tau$ represents derivation of $\tau, \hat{\omega}(\tau)$ and $\hat{A}(\tau)$ are the estimated value of frequency and amplitude respectively, $\hat{\omega}(\tau)>0 . K_{e}$ and $K_{f}$ are parameters that determine the accuracy of the frequency estimation and the rate of convergence respectively.

For convenience, choose a sinusoidal signal as input :

$$
y(\tau)=2 \sin \left(\omega_{0} \tau+0.4 \pi\right)
$$

The initial value $\omega_{0}$ is $1 \mathrm{~Hz}$ which jumps to $0.1 \mathrm{~Hz}$ at $\mathrm{t}=50 \mathrm{~s}$.

Choose two sets of parameter values: $K_{e}=K_{f}=1, K_{e}=K_{f}=0.5$ and build simulink model to simulate according to the state space equation. Simulation results as flows.

From figure 1 and figure 2 , we can notice that the robustness of the system will be obiviously different when choose different parameters. The system is unstable and divergent when the frequency changes from $1 \mathrm{rad} / \mathrm{s}$ to $0.5 \mathrm{rad} / \mathrm{s}$ at $K e=K f=1$. However, when the other parameters are selected, the system is stable and convergent even if the frequency is smaller. We assume that the frequency probably has an influence on the parameters selection of the AIM algorithm 
system.

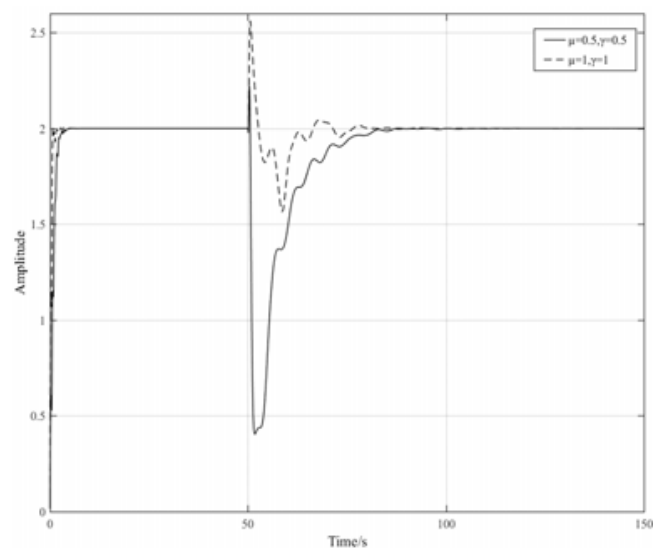

FIGURE I. AMPLITUDE FOLLOWING RESULTS OF DIFFERENT PARAMETERS BASED ON ADAPTIVE INTERNAL MODEL ALGORITHM

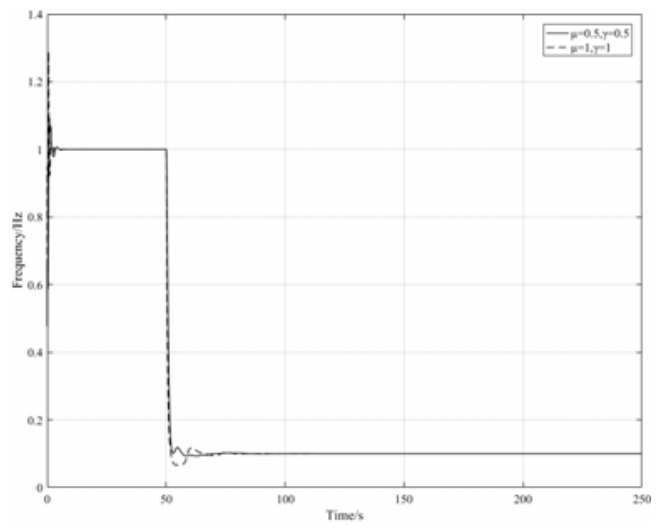

FIGURE II. FREQUENCY FOLLOWING RESULTS OF DIFFERENT PARAMETERS BASED ON ADAPTIVE INTERNAL MODEL ALGORITHM

In practical, we often deal with the tracking of frequency unknown signals and noise elimination. It can be seen that when the frequency is unknown, the accuracy of the model parameter selection is difficult, and multiple attempts are required. Therefore, we propose a new internal model algorithm solve the problem.

\section{A BI-NORMALIZED FREQUENCY ESTIMATION ALGORITHM}

According to the above simulation results, the relationship between the parameters $K e, K f$ and frequency in the internal model algorithm proposed in [7] is existed. In order to eliminate the influence of frequency on parameter selection, the following algorithm is proposed:

$$
\left\{\begin{array}{l}
\hat{x}_{1}^{\prime}(\tau)=-\widehat{\omega}(\tau) \widehat{x}_{2}(\tau) \\
\hat{x}_{2}^{\prime}(\tau)=\hat{\omega}(\tau)\left(\widehat{x}_{1}(\tau)+\mu\left(y(\tau)-\widehat{x}_{2}(\tau)\right)\right) \\
\widehat{\omega}^{\prime}(\tau)=\gamma \widehat{\omega}^{2}(\tau) \frac{\widehat{x}_{1}(\tau)\left(y(\tau)-\widehat{x}_{2}(\tau)\right)}{\hat{x}_{1}^{2}(\tau)+\hat{x}_{2}^{2}(\tau)}
\end{array}\right.
$$

The third of formula (5) is frequency update law. $\widehat{\omega}$ is estimated frequency, $\mu$ and $\gamma$ determine the accuracy of the frequency estimation and the rate of convergence respectively.

Next, analysis the stability of the proposed binormalization algorithm. Let $t=\omega_{0} \tau_{0}+\delta_{0}, d t=\omega_{0} d \tau_{0}$, then $\tau$ domain transform to $t$ domain and normalize the amplitude.The state equation of $t$ domain as follows:

$$
\left\{\begin{array}{l}
\dot{x}_{1}=-\theta x_{2} \\
\dot{x}_{2}=\theta\left(x_{1}+\mu\left(\sin t-x_{2}\right)\right) \\
\dot{\theta}=\gamma \theta^{2} x_{1}\left(\sin t-x_{2}\right)\left(x_{1}^{2}+x_{2}^{2}\right)^{-1}
\end{array}\right.
$$

In reference[4],let $\varepsilon=\sqrt{\gamma}$, errors define as:

$$
\boldsymbol{e}=\left[\begin{array}{l}
e_{1} \\
e_{2} \\
e_{3}
\end{array}\right]=\left[\begin{array}{c}
x_{1}-\cos t \\
x_{2}-\sin t \\
\varepsilon^{-1}(\theta-1)
\end{array}\right]
$$

Then error equation can be rewritten as a disturbance system:

$$
\dot{\boldsymbol{e}}=\boldsymbol{A}_{1}(t) e+\varepsilon \boldsymbol{g}_{1}(t, \boldsymbol{e})+\varepsilon \boldsymbol{g}_{2}(t, \boldsymbol{e})
$$

In which,

$$
\begin{gathered}
A_{1}=\left[\begin{array}{ccc}
0 & -1 & 0 \\
1 & -\mu & 0 \\
0 & 0 & 0
\end{array}\right], \boldsymbol{g}_{1}(t, \boldsymbol{e})=\left[\begin{array}{c}
-\sin t e_{3} \\
\cos t e_{3} \\
-\cos t e_{2}
\end{array}\right], \\
\boldsymbol{g}_{2}(t)=\left[\begin{array}{c}
-e_{2} e_{3} \\
-e_{3}\left(\mu e_{2}-e_{1}\right) \\
-\left(1+\varepsilon e_{3}\right)^{2}\left(e_{1}+\cos t\right) e_{2}+\cos t
\end{array}\right]
\end{gathered}
$$

In order to describe the stability of system (7),its linear periodic nominal system is considered:

$$
\dot{\boldsymbol{e}}=A_{1} \boldsymbol{e}+\varepsilon \boldsymbol{g}_{1}(t, \boldsymbol{e})
$$

The eigenvalue of matrix $\boldsymbol{A}_{1}$ are:

$$
\lambda_{1,2}=-0.5 \mu \pm 0.5 \sqrt{\mu^{2}-4}, \lambda_{3}=0
$$

And the perturbation $g_{1}(t, e)$ is continuous bounded and has a continuous first derivative. According to theorem VII 7.1 in [15], exists a constant $\varepsilon_{0}>0$,for fixed $\varepsilon$ which range in $0 \leq \varepsilon \leq \varepsilon_{0}$,there is always an integral 
manifold: $S_{\varepsilon}=\left\{(e, \boldsymbol{x}): e_{1}=\breve{e}_{1}(t, \varepsilon), e_{2}=\breve{e}_{2}(t, \varepsilon)\right\}$

And satisfy:

$$
\begin{aligned}
& \breve{e}_{1}(t, 0)=0, \breve{e}_{1}(t+2 \pi, \varepsilon)=\breve{e}_{1}(t, \varepsilon) \\
& \breve{e}_{2}(t, 0)=0, \breve{e}_{2}(t+2 \pi, \varepsilon)=\breve{e}_{2}(t, \varepsilon)
\end{aligned}
$$

In addition, the third equation of formula (8) derived again can get:

$$
\ddot{e}_{3}+\varepsilon^{2} \cos ^{2} t e_{3}=\varepsilon(\mu \cos t+\sin t) e_{2}-\varepsilon \cos t e_{1}
$$

On the integral manifold $S_{\varepsilon}$, let $e_{1}=e_{2}=0$ :

$$
\ddot{e}_{3}+\left(0.5 \varepsilon^{2}+0.5 \varepsilon^{2} \cos 2 t\right) e_{3}=0
$$

According to the reference [1] ,the near necessary condition for the stability of the system can be written as $\varepsilon^{2}=\gamma<2$.

In addition, according to the theorem 4.14 of the literature [14], it can be seen that if the exponential stable equilibrium point of the system (8) is $e=0$, there is a sufficiently small $\varepsilon$, make $e=0$ is also the exponential stable equilibrium point of the system (6).

So there exists $0<\gamma^{*}<2$, when $0<\gamma<\gamma^{*}<2$,

from algorithm (5) and formula (3) there are:

$$
\begin{gathered}
\widehat{x}_{1}(\tau) \rightarrow K_{0} \cos \left(\omega_{0} \tau+\delta_{0}\right), \quad \hat{\omega}(\tau) \rightarrow \omega_{0} \\
\hat{x}_{2}(\tau) \rightarrow y(\tau)=K_{0} \sin \left(\omega_{0} \tau+\delta_{0}\right) \\
\hat{A}(\tau)=\sqrt{\hat{x}_{1}^{2}(\tau)+\hat{x}_{2}^{2}(\tau)} \rightarrow K_{0}
\end{gathered}
$$

Compare algorithm (2) and (5) gets

$$
K_{f}=\mu \hat{\omega}(\tau), K_{e}=\gamma \omega(\tau) / \mu
$$

So the necessary condition for the convergence of algorithm (2) is $K_{e} K_{f}<2 \omega_{0}^{2}$. Observe figure 1 and figure 2, $K_{e}=K_{f}=1, \omega_{0}=1 \mathrm{~Hz}=2 \pi \mathrm{rad} / \mathrm{s}, K_{e} K_{f}=1<2 \omega_{0}^{2}=8 \pi^{2}$, the system meets the above necessary condition while $\omega_{0}=0.1 \mathrm{~Hz}=0.2 \pi$ $\mathrm{rad} / \mathrm{s}, K_{e} K_{f}=1>2 \omega_{0}^{2}=0.08 \pi^{2}=0.79$, it does not satisfy the condition.So the curves corresponding to $K_{e}=K_{f}=1$ in Figure 1 and Figure 2 do not converge when $\omega_{0}=0.1 \mathrm{~Hz}$.

\section{Simulation}

In order to verify the effective of the proposed algorithm,build simulation model according to (5) and (3) in MATLAB/Simulink, then make simulation experiment on the signal of formula(4).In order to ensure the comparability with the adaptive internal model algorithm (2), the two sets of parameters are selected, and the simulation results are shown in Figure 3 and Figure 4.

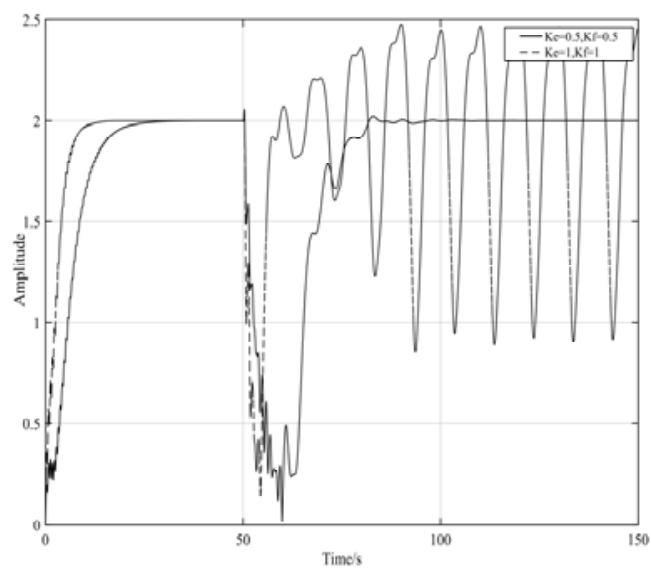

FIGURE III. AMPLITUDE FOLLOWING RESULTS OF DIFFERENT PARAMETERS BASED ON BI-NORMALIZED ALGORITHM

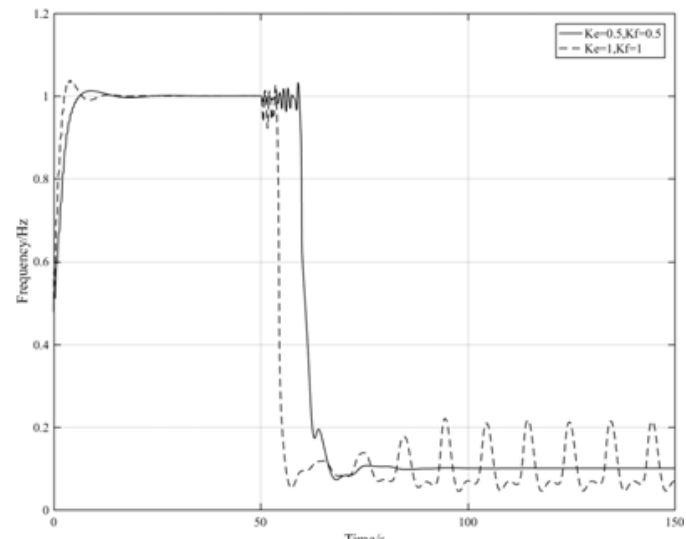

FIGURE IV. FREQUENCY FOLLOWING RESULTS OF DIFFERENT PARAMETERS BASED ON BI-NORMALIZED ALGORITHM

Compare Figure 1 and Figure 3, Figure 2 and Figure 4 respectively, we can find that the proposed algorithm still holds asymptotic convergence in small frequency conditions. Its convergence speed is faster and the overshoot is smaller which shows better robustness.

\section{CONCLUSION}

This paper proposed a bi-normalized frequency estimation algorithm based on the adaptive internal model algorithm which make the frequency estimation convergence is no longer related to the amplitude and frequency of the estimated signal. The convergence of the algorithm is illustrated. The simulation results show that the proposed algorithm still can follow the amplitude and frequency of the signal quickly even if the estimated frequency value is small, improve the robustness of the algorithm and extend the applicable range of the algorithm. 


\section{REFERENCES}

[1] Hsu L, Ortega R, Damm G. A globally convergent frequency estimator. IEEE Trans Autom Control 1999;44: 698-713

[2] Mojiri M,Bakhshai AR.An adaptive notch filter for frequency estimation of a periodic signal. IEEE Trans Autom Control 2004; 49: 314-318

[3] Chu Zhaobi,Zhang Chongwei,FengXiaoying.Adaptive notch filter-based frequency and amplitude estimation[J]. ActaAutomaticaSinica, 2010, 36(1): 60-66(in Chinese).

[4] Mojiri M, Bakhshai AR. Stability analysis of periodic orbit of an adaptive notch filter for frequency estimation of a periodic signal. Automatica 2007; 43: 450-455

[5] M. Ghadiri-Modarres,M. Mojiri, M. Karimi- Ghartemani, "New adaptive algorithm for delay estimation of sinusoidal signals with unknown frequency", IEEE Trans. Instrum. Meas., vol. 64, no. 9, pp. 2360-2366, Sep. 2015.

[6] Dai Wenzhan,Ding Liang,Yang Aiping.An Overview of the Development Of Internal Model Control[J]. Control Engineering of China, 2011(4).

[7] L. J. Brown and Q. Zhang,"Periodic disturbance cancellation with uncertain frequency," Automatica, vol. 40, no. 4, pp. 631-637, Apr. 2004.

[8] Zhang Q, Brown LJ.Noise analysis of an algorithm for uncertain frequency identification. IEEE Trans Autom Control 2006; 51: 103-110.

[9] M. K. Ghartemini,"Linear and pseudolinear enhanced phased-locked loop (EPLL) structures", IEEE Trans. Ind. Electron., vol. 61, no. 3, pp. 1464-1474, Mar. 2014

[10] G. Fedele, A. Ferrise,D. Frascino, "Structural properties of the SOGI system for parameters estimation of a biased sinusoid", Proc. 9th Int Conf. Environment and Electrical Engineering (EEEIC), pp. 438-441, 2010-May.

[11] M. Ciobotaru, R.Teodorescu, and F. Blaabjerg,"A new single-phase PLL structure based on second order generalized integrator", in Con] Rec. IEEE PESC, pp.I-6, Jun. 2006.

[12] Chu Zhaobi,DingMing,DuShaowu,et a1.Stability analysis of normalized frequency adaptive comb filter[J].Acta Autometica Sinica,2010,36(11): 1581-1593.

[13] G. Yin,L. Guo,and X. Li, “An amplitude adaptive notch filter for gridsignalprocessing,” IEEE Trans. Power Electron., vol. 28, no. 6, pp. 2638-2641, Jun. 20

[14] HassanK.Khalil,ZhuYisheng,DongHui,LiZuozhou,etal.NonlinerSystems ,Third Edition[M]. BEIJING:Publishing House of Electronics Industry 2011,pp.108-112.

[15] JACK HALE,HouDingpei.Ordinary differential equations[M]. BEIJING Publishing House of People's Education,1980,pp.286-287 